\title{
CIF/IFC Silver Ring Ceremony Lakehead University Class of 2020
}

This message was to be delivered to the 2020 Lakehead University Graduating Class at the CIF/IFC Silver Ring Ceremony March $21^{\text {st }}$ in Thunder Bay by Dean Caron of the Lake of the Woods Section. Covid-19 changed all that.

\section{Graduates of the Class of 2020!}

A lthough I very much wish I could be A there in person to deliver this message face to face and that the formal Ring Ceremony was taking place, due to the current situation with the Covid-19 virus, our being together to celebrate is not possible at this time. Regardless this is a very special time! I'm deeply honored to be selected by my CIF/IFC peers (Northwestern Ontario Section) and your class to have this opportunity to provide this message to you. I graduated from the Lakehead University School of Forestry with a HBSc of Forestry in 1994-28 years ago! My wife Karri is a LU forestry graduate (1993), my youngest son (Jayme) is completing his second year in the forestry program and my oldest son Derian, like you, is one of the graduates from this year's program!

This message I wanted to present to you is a very special one and an emotional one, as it is about you, the school, the CIF/IFC, and the Silver Ring. It's about the CIF/IFC officially welcoming you to the profession of forestry and most importantly, encouraging you to practice your profession within the Institute's Code of Ethics. These ceremonies have been held for many decades and have become a long-standing tradition for CIF/IFC Silver Ring schools across Canada.

This is the story behind the Silver Ring It all started in a committee room in the Montreal Board of Trade building on March $12^{\text {th }} 1908$ (about a year or so after the Kenora Thistles won the Stanley Cup!), where a group of "foresters" from across the country met with the intention of creating a nationwide "professional" organiza- tion. Their objectives were to share and discuss technical subject matter relating to the practice of forestry and to cultivate an "esprit de corps" among members of the profession.

In those days there were very few foresters and some may have thought a forester was synonymous with being a lumberjack; in any case, following a great deal of discussion, the word engineer was substituted for the word forester and The Canadian Society of Forest Engineers was initiated. The name of the organization was later changed to the Canadian Institute of Forestry/l'Institut forestier du Canada in 1950, reflecting a change in perspective about forests and foresters. Since its inception in 1908, the Institute has been the official voice of Canadian "forest practitioners" across the country, representing foresters, forest technologists and technicians, ecologists, biologists, geographers, educators, scientists and many others with a professional interest in forestry.

The Institute's mission is to: provide national leadership in forestry; promote competence among forestry professionals; foster public awareness of Canadian and international forestry issues; and promote the true nature of the profession.

The Institute serves a significant role in forestry continuing education, outreach and connecting forest professionals across the country. Every year the CIF/IFC recognizes worthy forest professionals and practitioners with a variety of national awards.

In 1953, the CIF/IFC Vancouver Section decided that it should recognize graduating foresters from the UBC Faculty of Forestry and the first Silver Rings were presented to the 1954 graduating class. In 1967, as a Centennial project, the CIF/IFC national headquarters officially adopted the administration of the Silver Ring program and in 2001, a new Silver Ring was created for graduating forest resource technologists. As of 2019, some 800 silver rings are ordered annually.

The traditions of the Silver Ring, which are derived from the engineer's Iron Ring, are intended to be a welcoming gesture to the profession, an acknowledgement of a student's achievements and serve as a visual sign of the bond that exists among Canadian forestry practitioners; a bond that overrides differences in levels of responsibility, employment status, province of residence, and language. Over the years, the Silver Ring has also become a symbol of the CIF/IFC, its principles and what it stands for, as embodied in the Code of Ethics. The Code of Ethics are:

1. Responsibility to Maintain the Public Good including forest stewardship, public confidence, public understanding and public welfare and safety;

2. Responsibility to the Profession including improving the practice of forestry and ensuring competency;

3. Responsibility to the Employer or Client with consistent, professional and dedicated service, understanding the consequences of actions, maintaining confidentiality and avoiding conflict of interest; and

4. Responsibility to Other Professionals with fairness and support, and the reporting of questionable practice.

These ethics are paramount in your professional lives.

If this were a perfect world, I along with my friends from the Northwestern Ontario Section would be presenting each of you in person with your own CIF/IFC Silver Ring. Given the situation we are currently challenged with, this is not possible. I ask that you wear your CIF/IFC Silver Ring with pride and humility... your Silver Ring should symbolize your commitment to do everything ethically, and with professionalism, first and foremost. The Ring should never manifest in any way elitism, special status or exclusivity. 


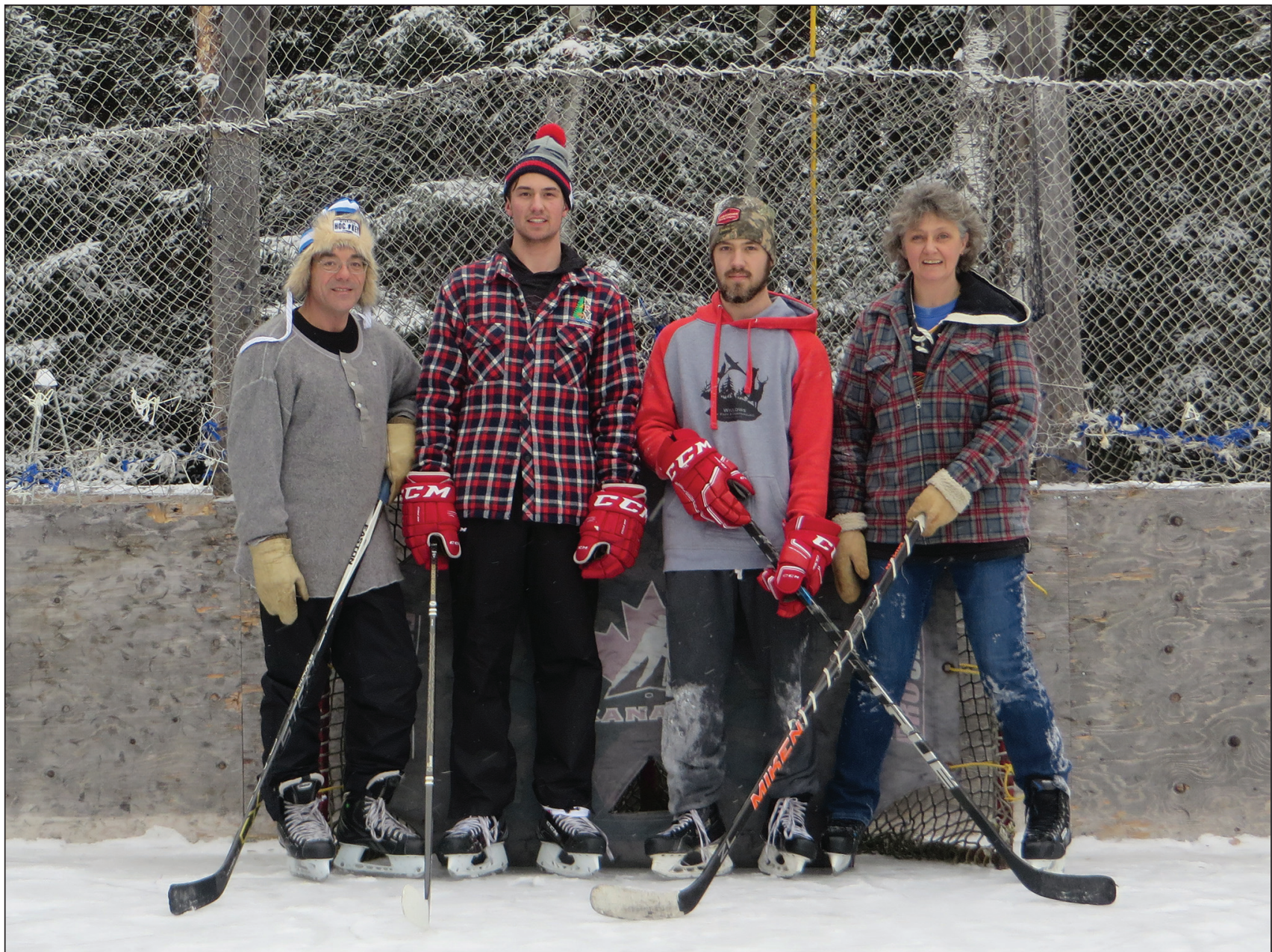

L to R: Dean Caron (1994 Lakehead graduate), Jayme (now 19 years old-20 in May, finished 2nd year at Lakehead Forestry), Derian (graduating this spring Lakehead Forestry 22 years old-working at Miisun in Kenora) and Karri Caron (1993 Lakehead graduate)/ G à D : Dean Caron (diplômé de Lakehead 1994), Jayme-20 ans en mai, étudiant de $2^{\text {ième }}$ année en Foresterie à Lakehead), Derian (diplômé de cette année en Foresterie à Lakeheademployé par Milsun de Kenora) et Karri Caron (diplômée de Lakehead 1993)

Wearing the Ring involves some very specific CIF/IFC traditions. It is placed on the little finger of your right hand-with the base of the leaf pointed toward your heart and the tip of the leaf pointing out-symbolizing the potential for growth within you... and of course your willingness to always grow. It symbolizes your commitment to lifelong learning, continuing education, professional development and most importantly, your commitment to live by the Code of Ethics of the Institute.

The Ring represents our commitment to work together within a broad and open society that recognizes the true interdisciplinary nature of forestry. Forestry has evolved from the original but still important economic focus on harvesting operations, productivity, growth and yield and silvicultural regeneration-to include wildlife habitat, biodiversity, soil and water conservation, and vital social and cultural themes, including the importance of forests to Indigenous people.

Modern forestry is far too complex for any one individual or professional to have all of the answers or all of the training-it takes a lot of different knowledge and expertise to even attempt to understand or manage all things forest-related.

Although the current situation we are facing in these troubling times is concerning, I'm confident that we as a society, our industry and us as foresters will emerge stronger from this crisis! We are foresters-we are used to fighting for what is right, we survive challenges and we emerge better from every challenge thrown at us! This current challenge is a big one, one we will overcome together as a society and be better for it!

I'm going to leave you with some advice to help you with your careers and life in general. This is not an exhaustive list, but rather the big hitters as I see them:

- Never forget your family and friends, always keep in touch, always remember it is because of them that you are what you are and who you are. My best friend at university became my wife and the mother of my children. She today continues to be my best friend. Without her I would not be here today (nor would my children!). She had a very profound and positive impact on my time at university and on my career, as did my parents and grandparents. 
Never forget your family and friends!

- Be humble and kind-treat people the way you want to be treated.

- Never wait for the "next time" to pass on kind words and let people know how you feel.

- Try and do random acts of kindness, everyday (make the world a better place).

- Safety first in everything you do (work and play), nothing we do is worth getting hurt for.

- Build networks, support the CIF/IFC, OPFA and other worthwhile organizations. Networks are critical. Take the time and effort to continually build yours.

- Be life-long learners, always ask questions (lots of questions)-you will earn respect for that; you can and will learn from many, many people over your career (that is a gift). Asking questions is a sign of strength. The best leaders and managers learn from everyone and ask lots of questions.

- Don't be afraid to take calculated risks. Trying new things and taking risks is how we continually improve.

- Remember, “you don't know what you don't know", don't be someone you are not, don't be afraid to admit you don't know something.

- Mistakes are gifts-because we can learn from them, often we learn more in failure than success. Don't be afraid of mistakes, always admit your mistakes and most importantly, learn from them.

- Challenges are a forester's specialty. In your career and in life you will be presented with many challengesprofessionally, personally and perhaps even in health. These challenges make us stronger (better people and professionals). As a profession I believe we are some of the strongest and best people out there! Accept any challenge with a positive attitude and can do/can succeed mentality (no matter what it is). Believe in yourself, believe in your teammates!

- No matter how tough things get or how big the challenges, always use your sense of humor! Even if you are not funny...

- Show appreciation for people that share knowledge with you.

- Knowledge is power-but only when shared. Share knowledge, always.

- Don't be afraid to try new things, work in new areas-stretch your comfort zone.

- Always offer solutions, never throw up barriers.

- As foresters, hold people accountable; do so with dignity and respect. Remember to "catch people doing good" far more often than "catching them doing bad".

- Remember data wins! Measure and quantify. Back up your position with facts and data.

- Your critics are your friends! If someone is critical of your work, listen to them, understand their position, although it may not be something you want to hear. If you listen and understand, you can likely make improvements and be even better!

- Try and put yourself in others' shoes, see things from their perspective to help you build and maintain relationships.

- Schools, industry, government, wood products/forestry support organizations-we are all here for the same reason: to support the industry. We need to remember that and work together as much as possible. We are at our best when we work together!

- Don't forget the folks that have taught you over the past four years. Some of these people even taught me (I'm old and they are even older...)! They are awesome people-you are not numbers at Lakehead University in the School of Natural Resources, you are people, often known by name to each and every one of your professors and lab helpers. These people that have shared their knowledge with you for the last four years care about you and your future; always remember them and keep in touch!

- Never discount any one thing as being small and insignificant-you never know what something will grow into and what the future holds. Twenty-eight years ago I would have never thought I would be providing the keynote address to the Lakehead Forestry class of 2020-with one of my children being a graduate! I'm a very proud parent, I'm proud of the staff of this fine school and of course, of all you graduates!

Over the past four years I have gotten to know a good number of you quite well; the future of the forest resource is in good hands! You are a great group of fine young men and women-the sky is the limit! Dream and think big! I can't wait to see what this group is going to accomplish in the coming years!

I would like to offer my sincere congratulations to all of you on behalf of the CIF/IFC. Welcome to the exciting, complex, fascinating, demanding, and ever-evolving profession and practice of forestry.

Thank you and congratulations to all the Silver Ring recipients!

Special thanks to Karen Saunders and Natasha Machado of the Institute for their help in providing me with details on the history of the CIF/IFC Silver Ring!

Dean Caron, RPF

Industry Advisor, FPInnovations Kenora, Ontario (now Woodlands Manager, Eacom Timber Co., Ear Falls, Ontario) 


\section{Cérémonie de remise des joncs de forestiers Université Lakehead, Promotion de 2020}

Ci-joint le texte de l'allocution qui devait être faite devant les diplômé(e)s de la promotion de 2020 de l'Université Lakehead lors de la cérémonie de remise des joncs de forestiers prévue le 21 mars à Thunder Bay par Dean Caron de la section Lake of the Woods. Les mesures sanitaires prises dans le cadre de la Covid-19 ont bouleversé le plan prévu].

\section{Chers diplômé(e)s de la promotion de 2020 ! \\ m ême si j'avais prévu être présent pour cette allocution lors de la céré-} monie de remise des joncs de forestiers, les mesures sanitaires prises à cause de la Covid-19 ont fait en sorte qu'il n'était plus possible de célébrer votre promotion. Néanmoins, il s'agit après tout d'un événement spécial pour lequel je suis très honoré d'avoir été choisi par mes collègues de l'IFC/CIF (section Nord-Ouest de l'Ontario) et les membres de votre promotion pour vous transmettre ce message. J'ai reçu mon diplôme de l'École de foresterie de l'Université Lakehead en 1994-il y a déjà 28 ans ! Mon épouse Karri est également diplômée en foresterie de l'UL (1993), mon plus jeune fils étudie actuellement en deuxième année de foresterie et mon fils aîné Derian, fait partie comme vous de la promotion de cette année!

Ce message que je voulais vous transmettre est à la fois spécial et émotif, car il s'agit de vous, de votre école, de l'IFC/CIF et du jonc de forestier. Il s'agit également de l'accueil officiel que l'IFC/CIF transmet à tous les diplômé(e)s en foresterie afin de vous encourager à pratiquer votre profession selon le code de déontologie de l'Institut. Ces cérémonies se sont déroulées depuis des décennies et font partie de la tradition séculaire de remise des joncs de forestiers partout au Canada.

Voici l'historique du jonc de forestier... Tout a débuté dans une salle de réunion de l'édifice de la Bourse de Montréal le 12 mars 1908 (près d'un an après que les Thistles de Kenora eurent remporté la
Coupe Stanley !) alors qu'un groupe de «forestiers » provenant de partout au pays se sont réunis dans le but de mettre en place une organisation "professionnelle » pour l'ensemble du pays. Leurs objectifs étaient de partager et de discuter de sujets techniques portant sur la pratique de la foresterie et d'entretenir un " esprit de corps » au sein des membres de la profession.

À cette époque il y avait très peu de forestiers et plusieurs personnes croyaient qu'un forestier était simplement un autre nom pour bûcheron, mais peu importe, à la suite de multiples échanges, le terme ingénieur remplaça le terme forestier et la Société d'ingénieurs forestiers du Canada débuta ses opérations. Le nom de l'organisation fut changé par la suite en 1950 pour la désignation de Canadian Institute of Forestry/Institut forestier du Canada afin de refléter le changement de perspective envers les forêts et les forestiers. Depuis sa création en 1908, l'Institut est le porte-parole officiel des professionnels de la foresterie du Canada, qu'ils soient forestiers, technologistes et techniciens forestiers, écologistes, biologistes, géographes, enseignants, chercheurs ou toutes autres personnes ayant un intérêt professionnel pour la foresterie.

La mission de l'Institut consiste à : assumer un leadership national en foresterie, promouvoir la compétence parmi les professionnels de la foresterie, sensibiliser l'intérêt public relativement aux enjeux forestiers du Canada et de la scène internationale et à promouvoir la vraie nature de la profession.

L'Institut joue un rôle significatif en matière de formation continue en foresterie et d'éducation et de diffusion parmi les professionnels de la foresterie au Canada. Chaque année, l'Institut souligne le travail exemplaire des professionnels de la foresterie en remettant plusieurs prix nationaux.

En 1953, la section Vancouver de l'Institut a décidé d'honorer les diplômés de la Faculté de Foresterie de l'UCB et les premiers joncs de forestiers furent remis aux diplômés de la promotion de
1954. En 1967, à titre de projet du Centenaire, le bureau de direction nationale de l'Institut a officiellement adopté le programme des joncs de forestiers et en 2001, un nouveau jonc a été conçu pour les diplômés en technologies des ressources forestières. En 2019, c'est près de 800 joncs de forestiers qui ont été remis.

La tradition rattachée au jonc de forestier, laquelle découle du jonc d'acier des ingénieurs, se veut un geste d'accueil au sein de la profession, une reconnaissance du parcours académique des étudiants et un signe visuel du lien qui unit les professionnels de la foresterie du Canada, un lien qui va audelà des différences en termes de responsabilité, de statut d'emploi, de province de résidence et de langue de travail. Au cours des années, le jonc de forestier est également devenu un symbole de l'Institut, de ses principes et de ce qu'il défend, tels que décrits dans son code de déontologie. Celui-ci se décrit ainsi :

1. La responsabilité du maintien du bien public ce qui inclus l'intendance des forêts, la confiance du public, la compréhension par le public ainsi que sa santé et sa sécurité;

2. La responsabilité envers la profession-ce qui inclus le développement de la pratique de la foresterie et de la compétence parmi les membres;

3. La responsabilité envers l'employeur ou le client par le maintien d'un service uniforme, professionnel et exclusif, par la compréhension des conséquences des gestes posés, par le maintien de la confidentialité et l'évitement de conflit d'intérêt et;

4. La responsabilité envers les autres professions par souci d'équité et de soutien et le signalement de pratiques douteuses.

Ce niveau de déontologie est primordial dans le cadre de nos vies professionnelles.

Si le monde était parfait, je serais en mesure de vous remettre au nom de 
l'Institut et en présence de mes ami(e)s de la section Nord-Ouest de l'Ontario vos joncs de forestiers. Étant donné la situation à laquelle nous sommes confrontés, cela n'est pas possible. Je vous demande de porter votre jonc de forestier avec fierté et humilité... votre jonc de forestier devrait être un symbole de votre engagement à accomplir toutes vos tâches de façon éthique et professionnelle avant toute chose. Le jonc ne doit jamais être un signe d'élitisme, de statut spécial ou d'exclusivité.

Le port du jonc fait référence à des traditions très spécifiques pour l'Institut. Il se porte sur l'auriculaire de votre main droite-la base de la feuille vers votre coeur et le bout de la feuille pointant vers l'avant-un symbole du potentiel de croissance en vous... et évidemment de la volonté de vous développer. Ce jonc symbolise votre engagement envers un apprentissage votre vie durant, envers la formation continue, le développement professionnel et le plus important votre engagement à agir selon le code de déontologie de l'Institut.

Ce jonc représente également votre engagement à travailler avec vos collègues au sein d'une communauté ouverte qui reconnait la vraie nature interdisciplinaire de la foresterie. Cette dernière a évolué depuis ses débuts, mais est encore économiquement axée sur les opérations de récolte, la productivité, la croissance et le rendement, ainsi que la régénération sylvicole - ce qui inclus l'habitat faunique, la biodiversité, la conservation des sols et de l'eau, en plus des enjeux sociaux et culturaux et de l'importance des forêts pour les peuples autochtones.

La foresterie moderne est de loin trop complexe pour qu'un individu ou une profession puisse avoir toutes les réponses ou toute la formation nécessaire-il faut plusieurs sources de connaissances et d'expertises pour tenter de comprendre ou d'aménager tout ce qui se rapporte aux forêts.

Même si la situation actuelle à laquelle nous faisons face en cette période trouble est d'une gravité sans précédent, je demeure confiant que nous, en tant que société, industrie et forestiers nous ressortirons encore plus forts de cette crise! Nous sommes des forestiers-nous avons l'habitude de nous battre pour ce qui est bien, nous surmontons les défis et nous sommes mieux équipés après chaque défi que nous affrontons! La situation actuelle est sans précédent, du genre que nous vaincrons en tant que société et pour être encore meilleurs !

J'aimerais vous donner quelques conseils afin de vous aider au cours de votre carrière et dans la vie en général. Il ne s'agit pas d'une liste exhaustive, mais plutôt des points marquants tels que je les voie :

- N’oubliez jamais votre famille et vos amis, restez en contact, souvenezvous que c'est pour eux que vous êtes ce que vous êtes. Ma meilleure amie du temps de l'université est devenue mon épouse et la mère de mes enfants. Et encore aujourd'hui elle demeure ma meilleure amie. Sans elle, je ne serais pas ici en ce jour (ni mes enfants !). Elle a eu un impact important et positif lors de mon passage à l'université et au cours de ma carrière, ainsi que mes parents et mes grands-parents. N'oubliez jamais votre famille et vos amis!

- Soyez humble et avenant-traitez les gens comme vous aimeriez être traité.

- N'attendez jamais à la prochaine fois pour transmettre des propos aimables et pour informer vos proches de ce que vous ressentez.

- Tâchez d'être bienveillant-chaque jour (faites en sorte que le monde soit un meilleur endroit pour $y$ vivre)

- La sécurité devrait être au cœur de vos préoccupations dans tout ce que vous faites (au travail ou dans vos loisirs), rien de ce qu'on fait ne vaut la peine de risquer des blessures.

- Élaborez vos réseaux, appuyez l'Institut, votre association professionnelle et toutes autres organisations significatives. Les réseaux sont essentiels. Prenez le temps et la peine de continuellement étendre les vôtres.

- N'arrêtez pas de vous perfectionner, poser souvent des questions (des tas de questions) - vous vous attirerez le respect des autres et vous allez pouvoir approfondir vos connaissances au contact des très nombreuses personnes que vous rencontrerez au cours de votre carrière (des occasions à saisir). Poser des questions est un signe de force. Les meilleurs chefs de file et les meilleurs gestionnaires apprennent au contact des autres et posent beaucoup de questions.

- N'ayez pas peur de prendre des risques calculés. L'essai de nouvelles choses et la prise de risque constituent une façon de continuellement s'améliorer.

- Souvenez que « vous ne savez pas ce que vous ignorez ", demeurez vousmême, n'ayez pas peur d'admettre que vos connaissances ont des limites.

- Les erreurs sont des opportunités parce qu'on peut en tirer des leçons, très souvent on en apprend plus de nos erreurs que de nos succès. N'ayez pas peur des erreurs, n'ayez crainte de vos erreurs, mais surtout tirez-en des leçons.

- Les défis sont une spécialité des forestiers. Au cours de votre carrière et de votre vie vous aurez à affronter de nombreux défis-professionnels, personnels et peut-être même médicaux. Ces défis nous rendent plus forts (en tant qu'êtres humains et en tant que professionnels). Dans le cas de notre profession, j'estime que nous formons un groupe de personnes parmi les plus fortes et les meilleures qui soient! Relevez tous les défis positivement et avec le sentiment que tout ira pour le mieux (peu importe de ce dont il s'agit). Ayez foi en vous-même et en vos collègues!

- Peu importe si les difficultés s'amoncellent ou si les défis deviennent de plus en plus importants, sachez utiliser votre sens de l'humour! Même si vous n'êtes pas drôle...

- Sachez remercier les gens qui vous font partager leurs connaissances.

- Le savoir est une force-mais seulement s'il est partagé. N'hésitez jamais à partager vos connaissances.

- N'ayez pas peur d'essayer de nouvelles choses, de travailler dans de nouveaux domaines-explorer les limites de votre zone de confort.

- Faites preuve d'ouverture, ne mettez pas de barrières.

- En tant que forestiers, considérez les gens comme étant responsables, faites-le avec dignité et respect. Souvenez-vous qu'il est mieux la plupart du temps de « surprendre les gens en train de bien faire les choses » que de les surprendre à mal les faire ".

- Souvenez-vous que les données sont des preuves! Mesurez et quantifiez. 
Appuyez votre position au moyen de faits et de données.

- Les critiques sont vos amis! Si une personne critique votre travail écoutez-la, prenez le temps de comprendre son point de vue, même s'il s'agit de quelque chose que vous ne voulez pas entendre. Grâce à votre écoute et à votre analyse, vous serez probablement en mesure d'améliorer votre travail et de le rendre encore meilleur!

- Essayez de vous mettre dans les souliers des autres-considérez les choses de leur point de vue afin de vous aider à établir et à maintenir vos relations.

- Les maisons d'enseignement, l'industrie, le gouvernement, les organisations connexes du secteur des produits forestiers et du monde de la foresterie sont tous là pour la même raison : appuyer l'industrie. Nous sommes à notre meilleur lorsque nous travaillons ensemble!

- N'oubliez pas les personnes qui vous ont enseigné au cours des quatre dernières années. Certaines d'entre elles m’ont également enseigné (je suis vieux et elles sont encore plus âgées... !) Ce sont des gens extraor- dinaires-vous n'êtes pas des numéros à l'École des Ressources naturelles de l'Université Lakehead, vous êtes des personnes, souvent connues par votre nom par chacun des professeurs et des chargés de laboratoire. Ces personnes avec qui vous avez partagé leurs connaissances au cours des quatre dernières années se sont souciées de vous et de votre avenir, gardez-les dans vos souvenirs et maintenez les contacts !

- Ne considérez jamais que quelque chose peut être futile et insignifiante-vous ne savez jamais ce qu'il en adviendra et ce que l'avenir vous réserve. Je n'aurais jamais pensé il y a vingt-huit ans que je livrerais l'allocution principale à l'attention des diplômés de la promotion 2020 de Foresterie de Lakehead-et que l'un de mes enfants en ferait partie! Je suis fier de lui en tant que parent, je suis fier du personnel de cette honorable école et, bien sûr de tous les diplômé(e)s !

$\mathrm{Au}$ cours des quatre dernières années, j'ai eu l'opportunité de faire connaissance avec un bon nombre d'entre vous, l'avenir de la foresterie est dans vos mains ! Vous formez un magnifique groupe de jeunes personnes-il n'y a plus de limites! Pensez grand! J'ai hâte de voir ce que groupe accomplira au cours des prochaines années!

J'aimerais vous transmettre mes sincères félicitations au nom de l'Institut forestier du Canada. Nous vous souhaitons la bienvenue au sein de la profession et de la pratique de la foresterie qui de par sa nature est excitante, complexe, fascinante, exigeante et constamment en évolution.

Merci et félicitations aux récipiendaires du jonc de forestier !

J'aimerais spécialement remercier Karen Saunders et Natasha Machado de l'Institut pour leur aide au niveau des détails de l'historique du jonc de forestier de l'IFC/CIF !

Dean Caron, RPF

Conseiller industriel, FPInnovations Kenora, Ontario (Maintenant, Gestionnaire des terraines boisés, Eacom Timber Co., Ear Falls, Ontario) 